\title{
State-Space Model and PQ Operating Zone Analysis of Hybrid MMC
}

\author{
Xiaojun $\mathrm{Lu}^{1}$, Wang Xiang*1 ${ }^{1}$, Weixing Lin $^{2}$, Jinyu Wen ${ }^{1}$
}

1 State Key Laboratory of Advanced Electromagnetic Engineering and Technology, Huazhong University of Science and Technology, Wuhan 430074, China

2 TBEA China Xinjiang Sunoasis Co. Ltd., Xi’an 710000, China

Abstract - A steady-state time-invariant (SSTI) state-space model is proposed in this paper for the hybrid modular multilevel converter (hybrid MMC). To analyze the internal dynamics of the hybrid MMC, the phasor modelling method is employed with considering three different frequency components in modulation signals. Originating from the state-space model, the steady-state model is obtained for analytically illustrating operating characteristics of the hybrid MMC. A 'scan and check' method is proposed to determine the feasible $P Q$ operating zone of the hybrid $\mathrm{MMC}$ with considering multiple operating constraints, especially the requirement for successful voltage balancing of half-bridge sub-modules (HBSMs). The accuracy of the state-space model is verified by comparing the time-domain response with the equivalent electromagnetic model of a hybrid MMC in PSCAD/EMTDC. Feasible operating zones of the tested system under different DC terminal voltages are calculated. The effects of different operating constraints on limiting the $P Q$ operating zone are analyzed. The impact of converter parameters such as the sub-module ratio, the arm reactance and the sub-module capacitance on the operating zone is presented in detail.

Key Words-Analytical model, hybrid modular multilevel converter, small-signal analysis,
steady-state analysis, HVDC transmission

\section{INTRODUCTION}

The application of the modular multilevel converter (MMC) in practical voltage source converter based high voltage direct current (VSC-HVDC) transmission projects has drawn significant attention

\footnotetext{
*Corresponding author. Tel: +86 13476095768

E-mail address: (*xiangwang1003@ foxmail.com).
} 
due to the various advantages of MMC in operation and control[1]. Comprehensive studies on design, modelling and application of the half-bridge sub-module (HBSM) based MMC (HB-MMC) have been reported[2]-[7]. But the HB-MMC lacks the ability to isolate the DC fault current. To enable overhead line transmission, the full-bridge sub-module (FBSM) based MMC (FB-MMC) can be employed, which has the capability to output both positive and negative voltages and actively interrupt the DC fault current. However, the high cost and power loss hamper the application of the FB-MMC. To achieve a trade-off between DC fault isolation ability and economic competitiveness, the MMC composed with mixed HBSMs and FBSMs (termed as the hybrid MMC) is considered as an effective approach [8]-[14].

Recently, there has been increasing attention on the topology, sub-module ratio design and the control strategy of the hybrid MMC[8]-[14]. To analytically describe operating characteristics and analyze the small signal stability of the hybrid MMC, a steady-state time-invariant (SSTI) state-space model should be developed[15]. There have been some researches on the SSTI modelling of the HB-MMC[15]-[17]. These models are primarily derived based on average dynamics of the MMC with well representing the internal dynamics of the HB-MMC[18]. However, compared with the HB-MMC, the hybrid MMC can output negative voltage and operate under the reduced DC terminal voltage, which brings about more control freedoms and state variables. As a result, the state-space model of the HB-MMC cannot be directly applied to the hybrid MMC.

To enable small-signal and steady-state studies, this paper presented a generic SSTI model of the hybrid MMC using the phasor modelling method. The internal dynamics of the hybrid MMC are modelled under rotating frames, such as the dynamics of capacitor voltages and circulating currents. The difference of the SSTI models between the HB-MMC and the hybrid MMC is analyzed. By setting the derivatives of the SSTI model to be zeroes, a steady-state model is obtained. Based on the steady-state model, $P Q$ operating zones under different DC terminal voltages are portrayed using a 'scan and check' approach. A fast calculation method for approximately illustrating the $P Q$ operating zone regarding the HBSM balancing constraint is disclosed. The accuracy of the state-space model is 
validated by time-domain simulation in contrast with electromagnetic simulation.

The remainder of this paper is as follows. Section II demonstrates derivation of the SSTI state-space model of the hybrid MMC. Section III presents the steady-state model and the solving process based on the operating principle of the hybrid MMC. Section IV presents the proposed 'scan and check' calculation method as well as a fast calculation method for the hybrid MMC. Section V presents the simulation validation of the SSTI state-space model. Section VI presents the analysis of operating zones under different DC terminal voltages and the impact of operating constraints and converter parameters on the $P Q$ operating zone.

\section{Steady-State Time-Invariant State-Space Model of THE Hybrid MMC}

\section{A. Basic Operating Principle of the Hybrid MMC}

The equivalent circuit for one phase leg of the hybrid MMC is shown in Fig. 1. Each phase leg of the hybrid MMC is composed of half HBSMs and half FBSMs. Two controlled voltage sources $v_{\mathrm{p}}$ and $v_{\mathrm{n}}$ represent output voltages of the upper and lower arm respectively. $R_{\mathrm{arm}}$ and $L_{\mathrm{arm}}$ represent the equivalent resistance and reactance of the phase arm. $C_{\text {sub }}$ denotes the rated capacitance of the HBSM and FBSM. $i_{\text {diff, }} i_{\mathrm{p}}$ and $i_{\mathrm{n}}$ denote the circulating current, upper arm current and lower arm current respectively. Default positive directions of the currents are depicted as shown in Fig. 1. $u_{\mathrm{dc}}$ represents the DC terminal voltage. $i$ represents the AC current. $K_{\mathrm{T}}, R_{\mathrm{T}}$ and $L_{\mathrm{T}}$ are the ratio, equivalent resistance and equivalent reactance of the AC transformer. $R_{\mathrm{s}}$ and $L_{\mathrm{s}}$ are the equivalent resistance and reactance of the AC system. $u_{\mathrm{v}}, u_{\mathrm{p}}, u_{\mathrm{s}}$ are the voltages of the converter AC-side, the point of public coupling (PCC) and the AC system respectively. 


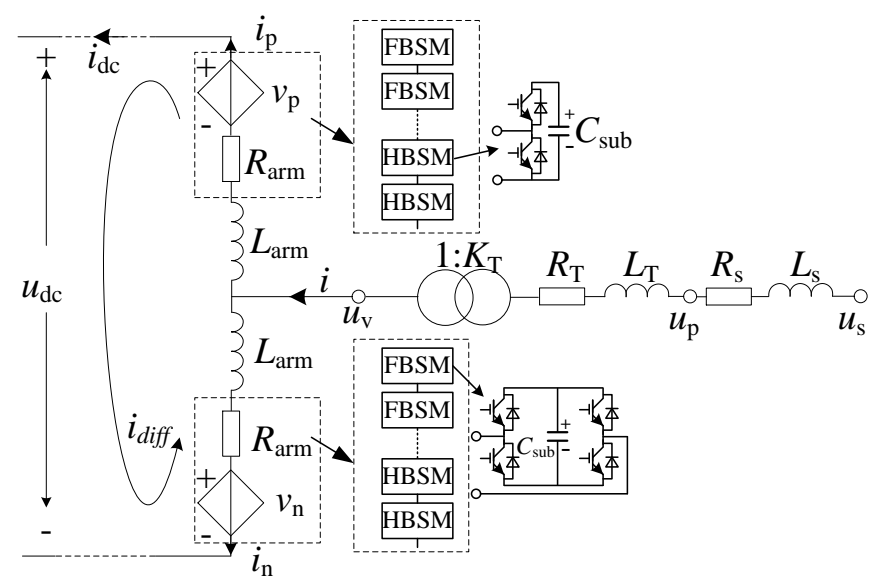

Fig. 1. Equivalent circuit for one phase leg of hybrid MMC

The elementary control scheme of the hybrid MMC[10] is illustrated in Fig. 2. Three modulation signals with different frequencies are generated by the elementary control. $m(\mathrm{t})$ generated by the inner AC current control loop is the modulation signal of fundamental frequency. The outer loop for $d$-axis regulates the average value $V_{\text {c,avg }}$ of total capacitor voltages in three phase arms to ensure the balance of arm energies. The outer loop for $q$-axis regulates the reactive power $Q_{\mathrm{pu}} m_{\mathrm{dc}}(\mathrm{t})$ is the DC modulation signal generated by the DC current control loop. The outer loop for the DC current control regulates the active power or the DC voltage and outputs the DC current reference value. $m_{\text {diff }}(\mathrm{t})$ generated by circulating current suppression control (CCSC) is the modulation signal of double fundamental frequency. This paper focuses on the electric system modelling for the hybrid MMC, and the control dynamics are not involved. The effect of the controller is embodied in the modulation signals, which are regarded as parameters for the open-loop state-space model.

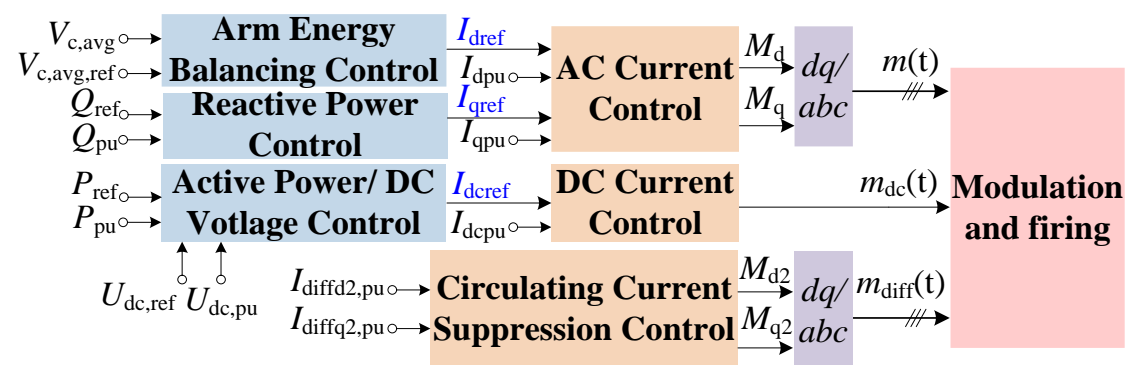

Fig. 2. Elementary control of a hybrid MMC

From the perspective of the phasor modelling, electric phasors for the electric system of the HB-MMC and hybrid MMC are similar. While according to Fig. 2, comparing the traditional double-loop control scheme of the HB-MMC with the elementary control scheme of the hybrid MMC, it 
can be found that the elementary control of the hybrid MMC introduces more control freedoms and constraints, which can be reflected in the composition of the arm modulation signals and the operating constraints.

Based on the analysis above, the difference of the phasor models for the HB-MMC and the hybrid MMC exists in the embodiment of the control system, i.e., the parameters of modulation signals. The modulation signals for the HB-MMC model in [16] need to be modified according to Fig. 2, while the rest parts in the HB-MMC model can be successively employed for the hybrid MMC model.

\section{B. State-Space Model of the Hybrid MMC}

Suppose that the rated DC terminal voltage of the hybrid MMC is $u_{\mathrm{dcN}}$, and the rated capacitor voltage of the HBSM and FBSM is $u_{\mathrm{cN}}$. Then the total number $N_{\mathrm{sm}}$ of sub-modules per arm can be calculated (neglecting redundancy) by

$$
N_{\mathrm{sm}}=u_{\mathrm{dcN}} / u_{\mathrm{cN}}
$$

Referring to the elementary control of the hybrid MMC, the modulation signals for the upper and lower arm $m_{\mathrm{p}}$ and $m_{\mathrm{n}}$ can be represented as

$$
\begin{aligned}
& m_{\mathrm{p}}(t)=\frac{1}{2} m_{\mathrm{dc}}(t)-\frac{1}{2} m(t)-\frac{1}{2} m_{\text {diff }}(t) \\
& m_{\mathrm{n}}(t)=\frac{1}{2} m_{\mathrm{dc}}(t)+\frac{1}{2} m(t)-\frac{1}{2} m_{\text {diff }}(t)
\end{aligned}
$$

As can be seen, compared with the HB-MMC, each arm modulation signal of the hybrid MMC contains a DC component $m_{\mathrm{dc}}(\mathrm{t})$ to regulate the DC voltage in order to match the reduced DC terminal voltage.

The output voltages of the upper and lower arm $v_{\mathrm{p}}(\mathrm{t})$ and $v_{\mathrm{n}}(\mathrm{t})$ can be expressed as[16]

$$
v_{\mathrm{p}}=m_{\mathrm{p}} v_{\mathrm{p}}^{\Sigma}, v_{\mathrm{n}}=m_{\mathrm{n}} v_{\mathrm{n}}^{\Sigma}
$$

where $v_{\mathrm{p}}^{\Sigma}$ and $v_{\mathrm{n}}^{\Sigma}$ denote the total capacitor voltage of the upper and lower arm respectively.

The circulating current $i_{\text {diff }}$ can be represented by arm currents as

$$
i_{\text {diff }}=\left(i_{\mathrm{p}}-i_{\mathrm{n}}\right) / 2
$$

Since the electric dynamics of the HB-MMC and hybrid MMC are similar, the SSTI state-space 
model of the hybrid MMC can be derived by directly replacing the modulation signals of the HB-MMC by (2). Thus, the single phase SSTI state-space model of the hybrid MMC under static ABC frame can be obtained as shown in (5), where $C_{\mathrm{arm}}=C_{\mathrm{sub}} / N_{\mathrm{sm}}$. The state variables $\left(v_{\mathrm{p}}^{\Sigma}, v_{\mathrm{n}}^{\Sigma}, i_{\text {diff }}\right)$ relate to the internal dynamics of the converter. The AC current and DC terminal voltage are regarded as electrical inputs.

$$
\begin{aligned}
& \frac{\mathrm{d}}{\mathrm{d} t}\left[\begin{array}{c}
v_{\mathrm{p}}^{\Sigma} \\
v_{\mathrm{n}}^{\Sigma} \\
i_{\mathrm{diff}}
\end{array}\right]=\left[\begin{array}{ccc}
0 & 0 & -\frac{m_{\mathrm{dc}}-m-m_{\mathrm{diff}}}{2 C_{\mathrm{arm}}} \\
0 & 0 & -\frac{m_{\mathrm{dc}}+m-m_{\mathrm{diff}}}{2 C_{\mathrm{arm}}} \\
\frac{m_{\mathrm{dc}}-m-m_{\mathrm{diff}}}{4 L_{\mathrm{arm}}} & \frac{m_{\mathrm{dc}}+m-m_{\mathrm{diff}}}{4 L_{\mathrm{arm}}} & -\frac{R_{\mathrm{arm}}}{L_{\mathrm{arm}}}
\end{array}\right]\left[\begin{array}{c}
v_{\mathrm{p}}^{\Sigma} \\
v_{\mathrm{n}}^{\Sigma} \\
i_{\mathrm{diff}}
\end{array}\right]+\left[\begin{array}{c}
-\frac{m_{\mathrm{dc}}-m-m_{\mathrm{diff}}}{4 C_{\mathrm{arm}}} \\
\frac{m_{\mathrm{dc}}+m-m_{\mathrm{diff}}}{4 C_{\mathrm{arm}}} \\
0
\end{array}\right] i+\left[\begin{array}{c}
0 \\
0 \\
\frac{-1}{2 L_{\mathrm{arm}}}
\end{array}\right] u_{\mathrm{dc}}
\end{aligned}
$$

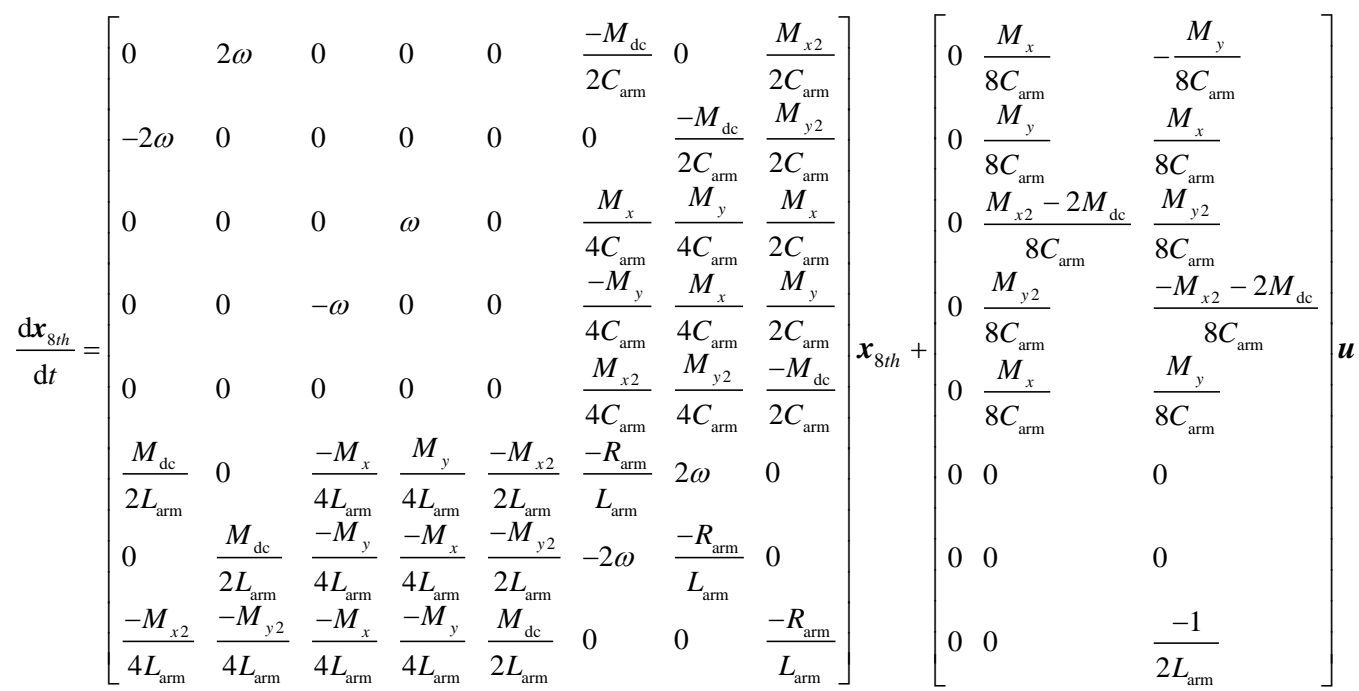

Since the state-space model of (5) is built in static ABC frame, the state variables and cells in the matrices contain variables with multiple frequencies. As a result, they are difficult to be analytically calculated. Based on the dynamic phasor modelling method proposed in [16][17] for the HB-MMC, the eight-order dynamic phasor model for the hybrid MMC under rotating frames can be obtained similarly, as shown in (6). The state-space model can be abbreviated as

$$
\dot{\boldsymbol{x}_{8 t h}}=\boldsymbol{A}_{8 t h} \boldsymbol{x}_{8 t h}+\boldsymbol{B}_{8 t h} \boldsymbol{u}
$$

where the state variable $\boldsymbol{x}_{8 \mathrm{th}}=\left[v_{\mathrm{p} x 2}^{\Sigma}, v_{\mathrm{p} y 2}^{\Sigma}, v_{\mathrm{p} x}^{\Sigma}, v_{\mathrm{p} y}^{\Sigma}, v_{\mathrm{p} 0}^{\Sigma}, i_{\mathrm{diff} x 2}, i_{\mathrm{diff} y 2}, i_{\mathrm{diff} 0}\right]^{\mathrm{T}}$, the input $\boldsymbol{u}=\left[u_{\mathrm{dc}}, i_{x}, i_{y}\right]^{\mathrm{T}}$. The subscript $x$ and $y$ denote the coordinate components of corresponding variables under the rotating frame with fundamental frequency. Similarly, the subscript $x 2$ and $y 2$ relate to the rotating frame with second harmonic frequency. The subscript 0 denotes the DC component. 
The elements in $\boldsymbol{A}_{8 \text { th }}$ include the electric parameters of the MMC such as $C_{\text {arm }}, R_{\text {arm }}, L_{\text {arm }}$ and the coordinate components of modulation signals indicated as $M_{\mathrm{dc}}, M_{x}, M_{y}, M_{x 2}$ and $M_{y 2}$. The steady-state values of the coordinate components of the modulation signals and electrical variables at a certain operating point are time-invariant, which makes (7) a SSTI state-space model. The small signal model of the hybrid MMC can be readily obtained by directly linearizing (7).

\section{STeady-State Model OF THE HybRId MMC}

\section{A. Steady-State Model of the Hybrid MMC}

By setting the derivatives of (7) to be zeroes, we can get the steady-state model of hybrid MMC, as represented in (8).

$$
\mathbf{0}=\boldsymbol{A}_{8 t h} \boldsymbol{x}_{8 t h}+\boldsymbol{B}_{8 t h} \boldsymbol{u}
$$

The steady-state model contains eight nonlinear algebraic equations. The detailed expressions for the algebraic equations will not be given since they can be easily obtained from (6). In the following context, ' 0 ' is added to the original subscripts of the variables to indicate steady-state values.

\section{B. Solving of the Steady-State Model}

The solving of the steady-state model can be converted to solving a group of nonlinear algebraic equations.

Apparently, the unknown variables of the steady-state model include eight electrical quantities and five control quantities, namely $v_{\mathrm{p} x 2}^{\Sigma}, v_{\mathrm{p} y 2}^{\Sigma}, v_{\mathrm{p} x}^{\Sigma}, v_{\mathrm{py}}^{\Sigma}, v_{\mathrm{p} 0}^{\Sigma}, i_{\text {diff } x 20}, i_{\text {diff } 20}, i_{\text {diff00 }}$ and $M_{\mathrm{dc} 0}, M_{x} 0, M_{y 0}, M_{x 20}, M_{y 20}$. However, the number of the unknown variables can be reduced by analysing the operating principle of

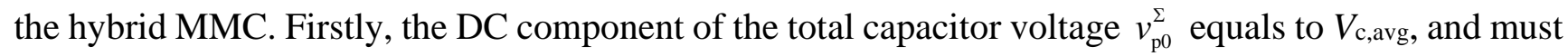
be controlled at its rated value to guarantee power balance, as shown in Fig. 2, which means that $v_{\mathrm{p} 0}^{\Sigma}$ equals to $u$ dcN at steady state. Secondly, when CCSC is enabled, $i_{\text {diff } x 20}$ and $i_{\text {diffy } 20}$ equal to zeroes; otherwise $M_{x 20}$ and $M_{y 20}$ equal to zeroes. So, it can be concluded that there are actually ten unknown variables in the steady-state model. While only eight equality constraints are obtained from the steady-state model, two more equality constraints are needed.

Depending on the conditions of a given operating point, different solutions can be derived 
accordingly. Suppose that the DC current $i_{\mathrm{dc} 0}$ and reactive power $Q_{0}$ are known variables at steady state, which indicates that hybrid MMC is under constant DC current and reactive power control, then we can get two equality constraints involved with $i_{\mathrm{dc} 0}$ and $Q_{0}$.

$$
\begin{gathered}
i_{\mathrm{dc} 0}=3 i_{\mathrm{diff} 0} \\
Q_{0}=\frac{3}{2}\left[\frac{u_{\mathrm{s} y 0} i_{x 0}-u_{\mathrm{sx} 0} i_{y 0}}{K_{\mathrm{T}}}-\frac{\omega\left(L_{\mathrm{s}}+L_{T}\right)\left(i_{x 0}^{2}+i_{y 0}^{2}\right)}{K_{\mathrm{T}}^{2}}\right]
\end{gathered}
$$

Since the coordinate frame ( $x y$ frame) for the electric system is positioned at phase A of the AC system voltage $u_{\mathrm{s}}, u_{\mathrm{s} y 0}$ equals to zero and $u_{\mathrm{s} x 0}$ equals to $U_{\mathrm{sm}}$ (the amplitude of the rated AC phase voltage). Notice that (10) introduces two more unknown variables $i_{x 0}$ and $i_{y 0}$. But they can be calculated according to the MMC's AC-side equivalent circuit, as shown in Fig. 3, where $e_{\mathrm{v}}$ is the AC voltage at converter side[16] $\left(e_{\mathrm{v}}=v_{\mathrm{p}}-v_{\mathrm{n}}\right)$.

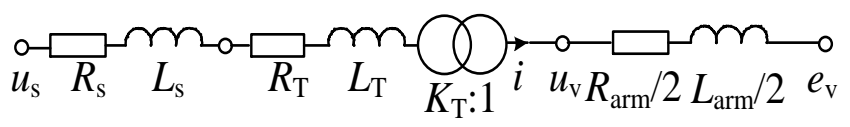

Fig. 3 Equivalent circuit of MMC AC-side

Based on KVL, the coordinate components for $e_{\mathrm{v}}$ can be represented with AC currents and AC system voltages as shown in (11), where $R_{\mathrm{tot}}=R_{\mathrm{arm}} / 2+\left(R_{\mathrm{T}}+R_{\mathrm{s}}\right) / K_{\mathrm{T}}^{2}, L_{\mathrm{tot}}=L_{\mathrm{arm}} / 2+\left(L_{\mathrm{T}}+L_{\mathrm{s}}\right) / K_{\mathrm{T}}^{2}$. According to the definition of $e_{\mathrm{v}}$, it can be represented by modulation signals and the total sub-module voltage as shown in (12), where $\boldsymbol{v}_{\mathrm{p}}^{\Sigma}=\left[v_{\mathrm{p} x 20}^{\Sigma}, v_{\mathrm{p} y 20}^{\Sigma}, v_{\mathrm{p} x 0}^{\Sigma}, v_{\mathrm{p} y 0}^{\Sigma}, v_{\mathrm{p} 00}^{\Sigma}\right]^{\mathrm{T}}$.

$$
\begin{gathered}
{\left[\begin{array}{l}
e_{\mathrm{v} x} \\
e_{\mathrm{vy} 0}
\end{array}\right]=\left[\begin{array}{cc}
-R_{\mathrm{tot}} & \omega L_{\mathrm{tot}} \\
-\omega L_{\mathrm{tot}} & -R_{\mathrm{tot}}
\end{array}\right]\left[\begin{array}{l}
i_{x 0} \\
i_{y 0}
\end{array}\right]+\left[\begin{array}{cc}
1 / K_{\mathrm{T}} & 0 \\
0 & 1 / K_{\mathrm{T}}
\end{array}\right]\left[\begin{array}{l}
u_{\mathrm{s} x 0} \\
u_{\mathrm{s} y 0}
\end{array}\right]} \\
{\left[\begin{array}{l}
e_{\mathrm{v} x 0} \\
e_{\mathrm{v} y 0}
\end{array}\right]=\left[\begin{array}{ccccc}
\frac{M_{x 0}}{4} & \frac{M_{y 0}}{4} & \frac{M_{x 20}-2 M_{\mathrm{dc} 0}}{4} & \frac{M_{y 20}}{4} & \frac{M_{x 0}}{2} \\
\frac{-M_{y 0}}{4} & \frac{M_{x 0}}{4} & \frac{M_{y 20}}{4} & \frac{-M_{x 20}-2 M_{\mathrm{dc} 0}}{4} & \frac{M_{y 0}}{2}
\end{array}\right] \boldsymbol{v}_{\mathrm{p}}^{\Sigma}}
\end{gathered}
$$

Equations (11) and (12) introduce two new unknown variables $e_{v x 0}$ and $e_{v y 0}$ and four equality constraints. In conclusion, there are fourteen unknown variables and fourteen equality constraints. By cooperatively solving (8)-(12), all the unknown variables can be solved.

Similarly, if the hybrid MMC operates under the active and reactive power control mode, we can replace (9) with the following equality constraint regarding the active power, 


$$
P_{0}=\frac{3}{2}\left[\frac{u_{\mathrm{s} x 0} i_{x 0}+u_{\mathrm{sy} 0} i_{y 0}}{K_{\mathrm{T}}}-\frac{\omega\left(R_{\mathrm{s}}+R_{T}\right)\left(i_{x 0}^{2}+i_{y 0}^{2}\right)}{K_{\mathrm{T}}^{2}}\right]
$$

The following calculation process is similar. By cooperatively solving (10)-(13), the unknown variables are calculated.

When the hybrid MMC operates under other control modes, we can obtain the initial values of the active power, the DC current or the reactive power through power flow calculation, and construct related equality constraints and finally solve the steady-state model by cooperatively solving the algebraic equations.

\section{PQ OPERATING ZONE OF THE HYBRID MMC}

The $P Q$ operating zone of a converter is one of the most concerned technical indices in practical projects during design stage. It can be used to check whether the parameters of a converter are qualified to make the converter cover the required range of active and reactive power. Since the converter should satisfy all kinds of operating constraints concerned with the electrical system and control system, it is essential to determine the feasible operating zone. By translating the operating constraints into mathematical expressions regarding the related electrical and control quantities, we can quantitatively analyse the feasible operating zone and the influence of different constraints on the operating zone through the steady-state model.

\section{A. Operating Constraints}

In [17], operating constraints regarding modulation signals, the arm current, the sub-module capacitor voltage ripple, the $\mathrm{AC}$ current and the $\mathrm{DC}$ current are considered. The mathematical expressions of the above operating constraints are reviewed here.

$$
\begin{gathered}
M_{\text {min }} \leq \sqrt{M_{x 0}^{2}+M_{y 0}^{2}} \leq M_{\text {max }} \\
\left|i_{\mathrm{p} 0}\right|_{\text {max }} \leq I_{\mathrm{arm}, \max },\left|i_{\mathrm{n} 0}\right|_{\max } \leq I_{\mathrm{arm}, \text { max }} \\
\left|\Delta u_{\mathrm{c} 0, \text { max }}\right| \leq \Delta U_{\mathrm{c}, \max } \\
\sqrt{i_{x 0}^{2}+i_{y 0}^{2}} \leq I_{\mathrm{ac}, \max } \\
I_{\mathrm{dc}} \leq I_{\mathrm{dc}, \max }
\end{gathered}
$$


As for hybrid MMC, once a HBSM is inserted, the arm current should contain both positive and negative components during one cycle to guarantee proper charging and discharging of HBSM capacitor. If the arm current contains only positive or negative component, the capacitor of inserted HBSM will keep charging or discharging, resulting in failed balancing of sub-module capacitor voltages. The requirement of bidirectional arm current can be mathematically expressed as

$$
\min \left\{i_{\mathrm{p} 0 \max }, i_{\mathrm{n} 0 \max }\right\}>0, \max \left\{i_{\mathrm{p} 0 \min }, i_{\mathrm{n} 0 \min }\right\}<0
$$

In this paper, the sorting and selection algorithm is not considered in detail to simplify the analysis. Suppose that the FBSM is recommended to be inserted first under reduced DC terminal voltage. Once the maximum value of the required arm output voltage is larger than the possible maximum aggregated voltage that all FBSMs are able to generate, extra HBSMs should be inserted to supplement the voltage gap. Referring to the upper arm, the maximum value of the upper arm voltage can be calculated by

$$
v_{\mathrm{p}, \max }=\max \left\{m_{\mathrm{p}} v_{\mathrm{p}}^{\Sigma}\right\}
$$

The maximum output voltage of the FBSMs can be approximated as $N_{\mathrm{FB}} * u_{\mathrm{cN}}$, where $N_{\mathrm{FB}}$ denotes the number of FBSM in one arm. Once (21) is satisfied, the HBSM should be inserted, then (19) should be satisfied.

$$
v_{\mathrm{p}, \max }>N_{\mathrm{FB}} u_{\mathrm{cN}}
$$

\section{B. The 'Scan and Check' Calculation Method for PQ Operating Zone}

To obtain the boundaries of the feasible $P Q$ operating zone considering multiple constraints, all the feasible operating points are needed to explore the boundaries. Since the feasible operating points can not be directly obtained from the constraints, they are produced in a 'scan and check' method. Considering that the hybrid MMC is able to operate under extreme small DC voltages, the corresponding active power is small due to the current constraints, which may bring challenges to the setting of the scanning range and varying step for active power. So the DC current and the reactive power are chosen to be the initial known conditions regarding the power operating level to calculate operating points. The procedures are detailed as follows:

Step 1: Determine the DC terminal voltage $u_{\mathrm{dc} 0}$; 
Step 2: Determine the primary searching area to be scanned according to the DC current constraint and the converter capacity constraint, which is $\left\{\left(i_{\mathrm{dc} 0}, Q_{0}\right) \mid-I_{\mathrm{dcmax}} \leq i_{\mathrm{dc} 0} \leq I_{\mathrm{dcmax}},-S_{\max } \leq Q_{0} \leq S_{\max }\right\}$, as shown in Fig. 4. Select an operating point $i_{\mathrm{dc} 0, \mathrm{k}}+\mathrm{j} Q_{0, \mathrm{k}}$ as the known conditions. Based on the steady-state model, the unknown variables including $i_{x 0}, i_{y 0}, \boldsymbol{x}_{8 t h 0}, M_{x 0}, M_{y 0}, M_{x 20}, M_{y 20}, M_{\mathrm{dc} 0}$ can be obtained;

Step 3: Check whether constraints (14)-(18) are satisfied. Calculate the maximum value of the arm output voltage, and check (21) to ascertain whether the HBSM needs to be inserted. If so, check(19).

Step 4: If all the constraints are satisfied, calculate the corresponding $P_{0, \mathrm{k}+1}+\mathrm{j} Q_{0, \mathrm{k}+1}$ and record this operating point. Select the next operating point $i_{\mathrm{dc} 0, \mathrm{k}+1}+\mathrm{j} Q_{0, \mathrm{k}+1}$ where $i_{\mathrm{dc} 0, \mathrm{k}+1}=i_{\mathrm{dc} 0, \mathrm{k}}, Q_{0, \mathrm{k}+1}=Q_{0, \mathrm{k}}+Q_{\mathrm{step}}$ or $i_{\mathrm{dc} 0, \mathrm{k}+1}=i_{\mathrm{dc} 0, \mathrm{k}}+I_{\mathrm{dc}, \mathrm{step}}, Q_{0, \mathrm{k}+1}=Q_{0, \mathrm{k}}$. Then, go back to Step 2 and check if this operating point is feasible.

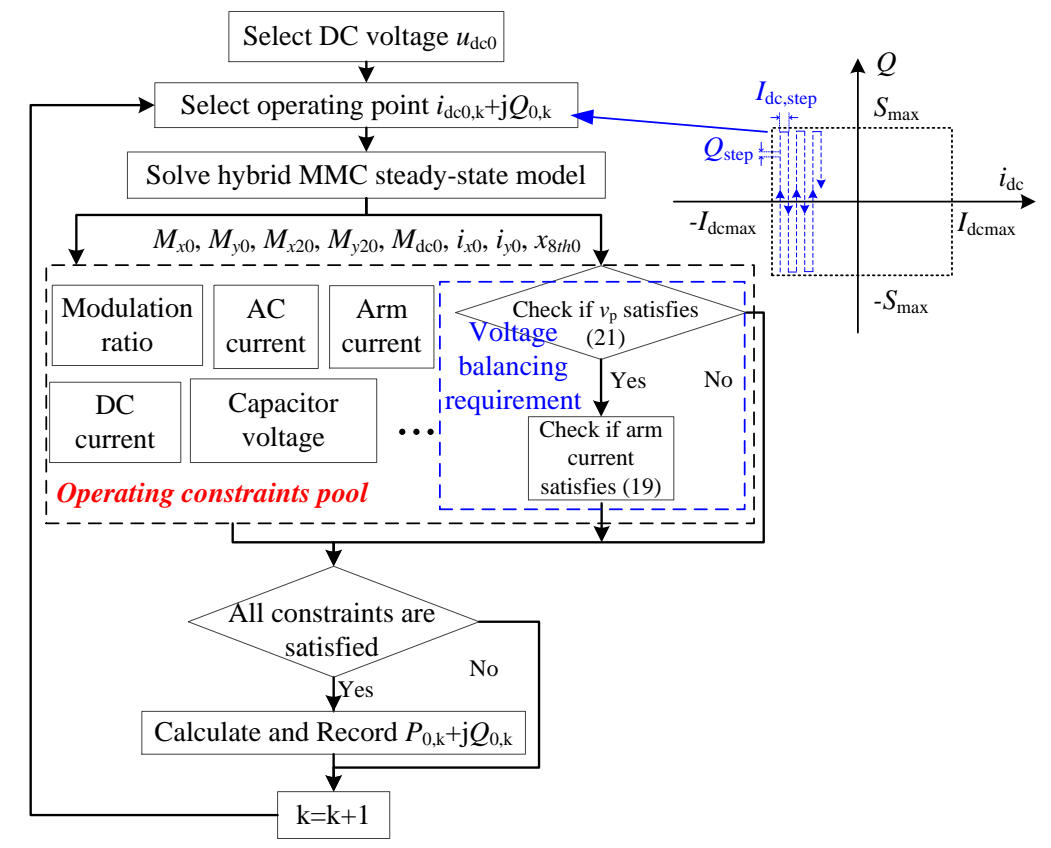

Fig. 4 Calculation procedure of the operating zone

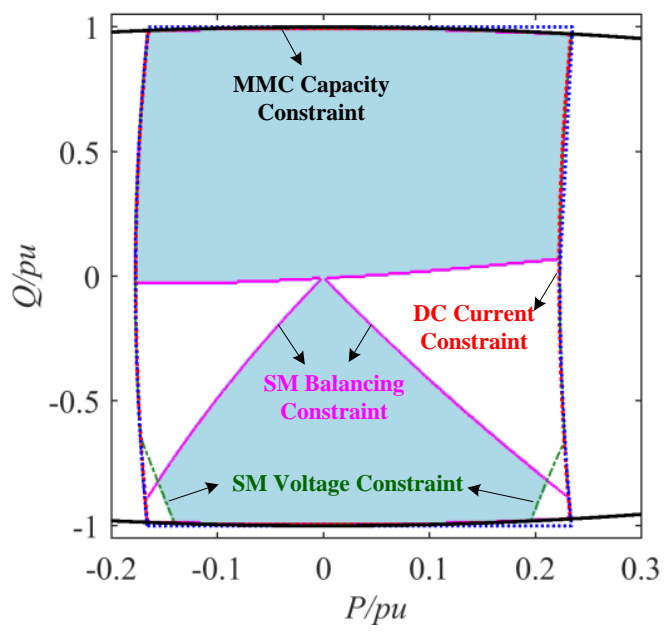

Fig. 5 The operating zone under 0.1 p.u. DC voltage 
By changing $u_{\mathrm{dc} 0}$, we can analyze the operating zone of hybrid MMC under different DC terminal voltages. To clarify the calculating procedures clearly, the flow chart of the calculation procedure is demonstrated in Fig. 4. An example of the calculation results for $P Q$ operating zone under 0.1 p.u. DC voltage is presented in Fig. 5. It can be observed that the irregular boundaries of the $P Q$ operating zone are mainly determined by the HBSM balancing constraint under the low DC terminal voltage.

\section{Fast Calculation Method of $P Q$ Boundaries Regarding the HBSM Balancing Constraint}

Notice that the 'scan and check' calculation method may be time-consuming since every possible operating point needs to be calculated. According to Fig. 5, the HBSM balancing constraint is dominant with contrast to other constraints in limiting the $P Q$ operating zone under reduced DC terminal voltages. Based on the requirement of arm currents and arm output voltages regarding the HBSM balancing constraint, it is better to develop a more efficient calculation method to estimate the approximated $P Q$ boundaries without losing too much accuracy.

\section{1) Fast Calculation Considering the Arm Current}

Taking the example of the upper arm current $i_{\mathrm{p}}$, it can be represented with the AC current and the circulating current as shown in (22). Since CCSC is enabled to eliminate $i_{\text {diff2, }} i_{\mathrm{p}}$ can be further simplified.

$$
\begin{aligned}
i_{\mathrm{p}} & =\frac{1}{2}\left(i_{x} \cos \omega t-i_{y} \sin \omega t\right)+i_{\mathrm{diff0}}+i_{\mathrm{diff} x 2} \cos 2 \omega t-i_{\mathrm{diff} y 2} \sin 2 \omega t \\
& =\frac{1}{2} \sqrt{i_{x}^{2}+i_{y}^{2}} \cos \theta_{i}+\frac{1}{3} i_{\mathrm{dc}}
\end{aligned}
$$

Considering (22), when the relation between the DC current and the AC current satisfies

$$
\left|\frac{i_{\mathrm{dc}}}{3}\right|=\frac{1}{2} \sqrt{i_{x}^{2}+i_{y}^{2}}
$$

the requirement for dual-direction of the arm current is critically fulfilled. If the power loss in the equivalent resistance $R_{\mathrm{s}}, R_{\mathrm{T}}, R_{\mathrm{arm}}$ and the reactance $L_{\mathrm{s}}, L_{\mathrm{T}}$ is neglected, the approximated active and reactive power of MMC $P_{\text {app }}$ and $Q_{\text {app }}$ can be calculated as 


$$
\begin{aligned}
& P_{\text {app }}=\frac{3}{2 K_{\mathrm{T}}}\left(u_{\mathrm{ss}} i_{x}+u_{\mathrm{ss}} i_{y}\right) \approx u_{\mathrm{dc}} i_{\mathrm{dc}} \\
& Q_{\mathrm{app}}=\frac{3}{2 K_{\mathrm{T}}}\left(u_{\mathrm{s} y} i_{x}-u_{\mathrm{s} x} i_{y}\right)
\end{aligned}
$$

Then $i_{x}$ can be approximated as shown in (25), where $K_{\mathrm{T}}=U_{\mathrm{sN}} / U_{\mathrm{vN}}, U_{\mathrm{sm}}=\sqrt{2} U_{\mathrm{sN}} / \sqrt{3}, U_{\mathrm{vN}}$ and $U_{\mathrm{sN}}$ are respectively the rated voltage at converter-side and grid-side of the transformer.

$$
i_{x} \approx \frac{2 u_{\mathrm{dc}} K_{\mathrm{T}}}{3 U_{\mathrm{sm}}} i_{\mathrm{dc}}=\sqrt{\frac{2}{3}} \frac{u_{\mathrm{dc}}}{U_{\mathrm{vN}}} i_{\mathrm{dc}}
$$

From (25), we can conclude that when $u_{\mathrm{dc}}>\sqrt{2} U_{\mathrm{vN}} / \sqrt{3}$, the DC and AC component in the arm current satisfies

$$
\frac{i_{\mathrm{dc}}}{3}<\frac{i_{x}}{2} \leq \frac{1}{2} \sqrt{i_{x}^{2}+i_{y}^{2}}
$$

which means the arm current definitely contains both positive and negative components.

When $u$ dc is smaller than $\sqrt{2} U_{\mathrm{vN}} / \sqrt{3}$, the $P Q$ boundaries limited by dual-direction requirement of the arm current can be obtained as below. Considering $u_{\mathrm{s} x}=U_{\mathrm{sm}}, u_{\mathrm{s} y}=0$, and combing (23) and (24), we can get the relation between $i_{x}$ and $i_{y}$, and also $P_{\text {app }}$ and $Q_{\text {app }}$ as

$$
\left|\frac{i_{x}}{i_{y}}\right|=\sqrt{\frac{K_{\mathrm{T}}^{2} u_{\mathrm{dc}}^{2}}{U_{\mathrm{sm}}^{2}-K_{\mathrm{T}}^{2} u_{\mathrm{dc}}^{2}}}=\left|\frac{P_{\text {app }}}{Q_{\mathrm{app}}}\right|
$$

From (27), an approximate linear relation between the active and reactive power exists when (18) is exactly satisfied, which means the $P Q$ boundaries determined by (18) are nearly straight lines in $P Q$ diagram of rectangular coordinate. The slope of the straight line is decided by the DC voltage. The areas between the two intersecting straight lines indicate the feasible operating zone from the perspective of dual-direction arm currents.

\section{2) Fast Calculation Considering the Arm Output Voltage}

When the required arm output voltage is smaller than $N_{\mathrm{FB}} * u_{\mathrm{CN}}$, it can be produced merely by FBSMs, whose voltages can be balanced regardless of the arm current direction. Applying KVL to the AC-side circuit of the hybrid MMC, and neglecting $R_{\mathrm{T}}$ and $R_{\mathrm{s}}, i_{x}$ and $i_{y}$ can be represented as 


$$
i_{x}=-\frac{K_{\mathrm{T}}^{2} u_{\mathrm{v} y}}{\omega\left(L_{\mathrm{s}}+L_{\mathrm{T}}\right)}, i_{y}=-\frac{K_{\mathrm{T}}\left(u_{\mathrm{s} x}-K_{\mathrm{T}} u_{\mathrm{v} x}\right)}{\omega\left(L_{\mathrm{s}}+L_{\mathrm{T}}\right)}
$$

The accurate active and reactive power of the converter can be calculated as

$$
P=\frac{3}{2}\left(u_{\mathrm{v} x} i_{x}+u_{\mathrm{v} y} i_{y}\right), Q=\frac{3}{2}\left(u_{\mathrm{v} y} i_{x}-u_{\mathrm{v} x} i_{y}\right)
$$

Insert (28) into (29), and we can obtain the expressions for $u_{\mathrm{v} x}$ and $u_{\mathrm{v} y}$,

$$
u_{\mathrm{v} x}=\frac{2 \omega\left(L_{\mathrm{s}}+L_{\mathrm{T}}\right)}{3 K_{\mathrm{T}} u_{\mathrm{s} x}} Q+\frac{K_{\mathrm{T}}\left(u_{\mathrm{v} x}^{2}+u_{\mathrm{v} y}^{2}\right)}{u_{\mathrm{s} x}}, u_{\mathrm{v} y}=-\frac{2 \omega\left(L_{\mathrm{s}}+L_{\mathrm{T}}\right)}{3 K_{\mathrm{T}} u_{\mathrm{s} x}} P
$$

From (30), the amplitude of $u_{\mathrm{v}}$ can be represented with $P$ and $Q$ as

$$
U_{\mathrm{vm}}^{2}=\left[\frac{2 \omega\left(L_{\mathrm{s}}+L_{\mathrm{T}}\right)}{3 K_{\mathrm{T}} u_{\mathrm{s} x}}\right]^{2}\left[P^{2}+\left(Q+\frac{3 K_{\mathrm{T}} U_{\mathrm{vm}}^{2}}{2 \omega\left(L_{\mathrm{s}}+L_{\mathrm{T}}\right)}\right)^{2}\right]
$$

where $U_{\mathrm{vm}}$ indicates the amplitude of $u_{\mathrm{v}}$. While from the converter-side, if we neglect the voltage across the equivalent arm reactance and resistance, $u_{\mathrm{v}}$ can be represented with $v_{\mathrm{p}}$ and $u_{\mathrm{dc}}$ as

$$
u_{\mathrm{v}}=\frac{1}{2} u_{\mathrm{dc}}-v_{\mathrm{p}}
$$

From (32), we can conclude that the possible range for $v_{\mathrm{p}}$ is $\left[-U_{\mathrm{vm}}+0.5 u_{\mathrm{dc}}, U_{\mathrm{vm}}+0.5 u_{\mathrm{dc}}\right]$. Suppose that only FBSMs are inserted to form the arm output voltage, the possible range for $v_{\mathrm{p}}$ is $\left[-N_{\mathrm{FB}} * u_{\mathrm{cN}}\right.$, $\left.N_{\mathrm{FB}} * u_{\mathrm{cN}}\right]$. If [- $\left.U_{\mathrm{vm}}+0.5 u \mathrm{dc}, U_{\mathrm{vm}}+0.5 u_{\mathrm{dc}}\right]$ is within [- $\left.N_{\mathrm{FB}} * u_{\mathrm{cN}}, N_{\mathrm{FB}} * u_{\mathrm{cN}}\right]$, i.e. $U_{\mathrm{vm}}$ satisfies

$$
\left\{\begin{array}{l}
U_{\mathrm{vm}} \leq N_{\mathrm{FB}} * u_{\mathrm{cN}}-0.5 u_{\mathrm{dc}}, u_{\mathrm{dc}}>0 \\
U_{\mathrm{vm}} \leq N_{\mathrm{FB}} * u_{\mathrm{cN}}+0.5 u_{\mathrm{dc}}, u_{\mathrm{dc}}<0
\end{array}\right.
$$

Then, the arm voltage can be produced merely by FBSMs. When $U_{\mathrm{vm}}$ reaches the boundary value of (33), we can obtain the qualified $P$ and $Q$ referring to (31). The boundary of the $P Q$ operating zone appears to be a circle with its center and radius decided by the DC terminal voltage and the FBSM ratio. The areas outside the circle indicate the feasible operating zone from the perspective of arm output voltages.

3) Comprehensive Analysis for PQ Boundaries Regarding the HBSM Balancing Constraint

Regarding the HBSM balancing constraint, equations (23) and (33) characterize boundaries of the $P Q$ operating zone from the perspective of arm currents and arm output voltages. If the operating point 
is located either between the straight lines calculated by (23) or outside the circle decided by (33), then it can be deemed to be feasible.

Taking the example of a hybrid MMC, whose parameters will be introduced in the next section, the boundaries produced by (23) and (33) under different DC voltage levels are shown in Fig. 6 (a)-(b). Notice that the converter capacity constraint $P^{2}+Q^{2} \leq 1$ is also considered, indicated as the unit circle. The comprehensive boundaries under each DC voltage are in bold. The corresponding feasible operating zones are shown in the shaded areas.

As can be seen, under low DC terminal voltage, the calculated boundaries have intersections within the unit circle, and the comprehensive boundaries are determined by the arm current and the arm output voltage together, as shown in Fig. 6 (a). With the rise of the DC voltage (see Fig. 6 (b)), the intersections of the calculated boundaries are outside the unit circle, and the comprehensive boundaries are decided by the dual-direction arm current only.

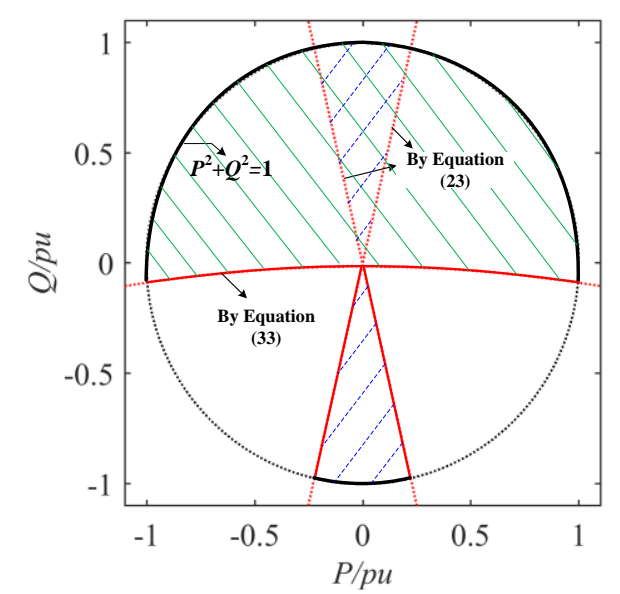

(a) 0.1 p.u. DC voltage

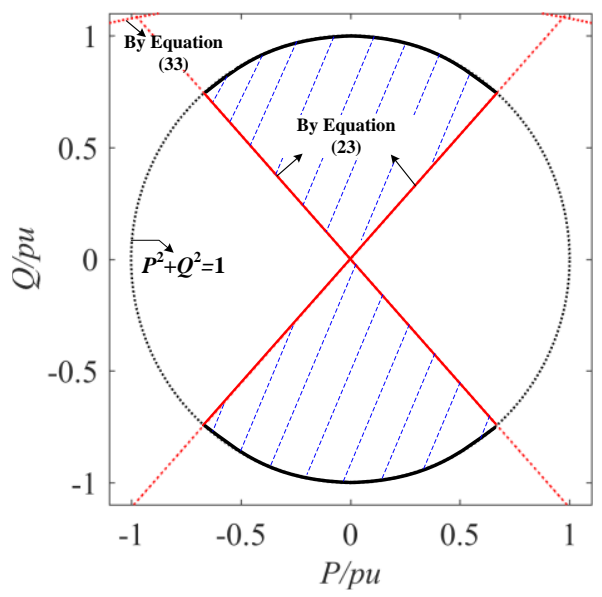

(b) 0.3 p.u. DC voltage

Arm Output Voltage Only by FBSMs

Dual-Direction Arm Current

Fig. $6 P Q$ boundaries characterized by HBSM balancing constraint under different DC voltages

Notice that the derivation of equations (23) and (33) contains much assumption, so the $P Q$ boundaries in Fig. 6 only provide rough estimation of the actual feasible $P Q$ operating zone characterized by the HBSM balancing constraint. To obtain the accurate boundaries of the feasible $P Q$ operating zone, the more elaborated 'scan and check' calculation method is recommended. 


\section{Simulation VAlidations}

\section{A. Test System}

The topology of the test system is shown in Fig. 7. The test system contains a single terminal hybrid MMC interfacing a controlled DC voltage source. The parameters of the test system are listed in Table 1. The output voltage of the DC voltage source can be adjusted to simulate different DC terminal voltages.

To verify the correctness of the SSTI state-space model, the simulation results obtained from the equivalent electromagnetic model[19] are compared with the analytical model. In the equivalent electromagnetic model, two controlled voltage sources represent the stacks of HBSMs and FBSMs separately in each arm. The equivalent electromagnetic model is able to capture the dynamics of each sub-module by considering the dynamic process of charging and discharging of sub-module capacitors[19].

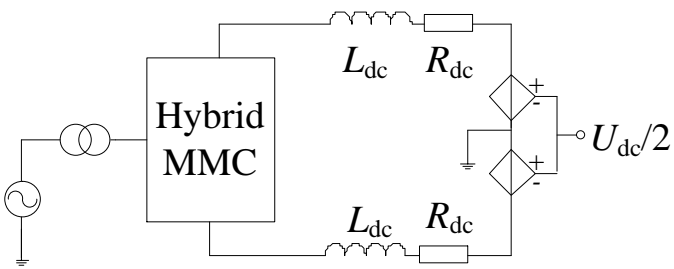

Fig. 7 Topology of the test system

Table 1 Parameters of the test system

\begin{tabular}{l|l|l}
\hline \multirow{2}{*}{ AC system } & Rated voltage & $525 \mathrm{kV}$ \\
& Equivalent impedance & $8.01 \Omega(\mathrm{X} / \mathrm{R}=7.87)$ \\
\hline \multirow{2}{*}{ AC } & Rated capacity & $900 \mathrm{MVA}$ \\
transformer & Ratio & $525 / 220$ \\
& Leak reactance & $0.15 \mathrm{p.u}$. \\
\hline \multirow{4}{*}{ Hybrid } & Rated capacity & $750 \mathrm{MVA}$ \\
$\mathrm{MMC}$ & Rated DC voltage & $400 \mathrm{kV}$ \\
& Number of sub-module & $95 / 95$ \\
& per arm(FB/HB) & $9 \mathrm{mF}$ \\
& $C_{\text {sub }}$ & $31 \mathrm{mH}$ \\
\hline DC line & $L_{\text {arm }}$ & $1.5 \Omega$ \\
\hline
\end{tabular}

\section{B. Verification of the State-Space Model}

The state-space model of the test system is built in Simulink/Matlab. The equivalent electromagnetic model of the test system is built in PSCAD/EMTDC. Constant DC current and reactive power control is employed in the test hybrid MMC. CCSC is enabled to suppress the second harmonic 
components in arm currents.

The simulation scenarios are stated as below:

(1) The DC voltage starts to decrease from the rated value to zero at $t=2.0 \mathrm{~s}$ with the velocity of -2 p.u./s.

(2) The DC current order increases from -1 p.u. to zero at $t=2.05 \mathrm{~s}$ with the velocity of 5 p.u./s.

(3) The reactive power order drops from -0.1 p.u. to -0.3 p.u. at $t=2.75 \mathrm{~s}$ with the velocity of -1 p.u./s.

Fig. 8 (a) shows the waveforms of the total capacitor voltage. From $2 \mathrm{~s}$ to $2.75 \mathrm{~s}$, with the decrease of the DC voltage, the AC components in the total capacitor voltage decay obviously. From $2.75 \mathrm{~s}$, with the increase of the output reactive power, the fluctuation in the total capacitor voltage rises. Fig. 8 (b) shows the waveforms of the arm current. The variation of the arm current is similar to that of the total capacitor voltage. Fig. 8 (c) shows the waveforms of the DC current. When the DC terminal voltage starts to decrease at $\mathrm{t}=2 \mathrm{~s}$, the DC current decreases to reduce $M_{\mathrm{dc}}$ in order to track the descending DC voltage. As the DC current order starts to reduce from $t=2.05 \mathrm{~s}$, the $\mathrm{DC}$ current tracks the decreasing order. Around $\mathrm{t}=2.5 \mathrm{~s}$, the transition of the controller ends and the DC current reaches steady state. From Fig. 8 (a)-(c), the good agreement of state-space model and the PSCAD model can be observed, which verifies the correctness of the proposed state-space model.

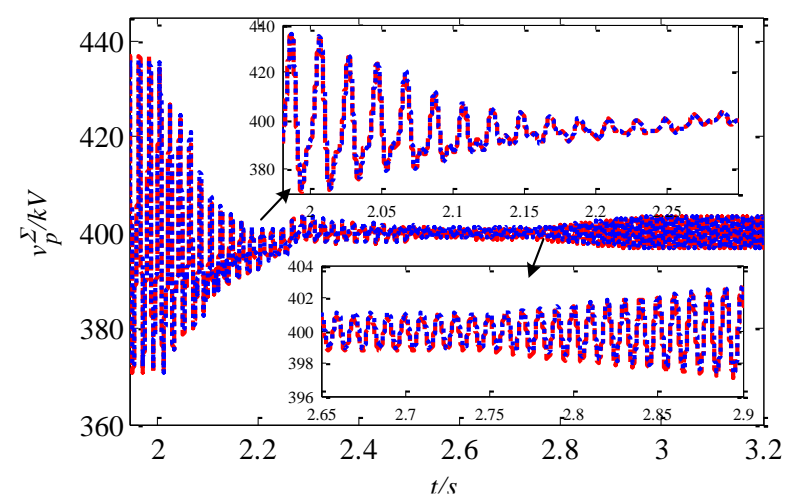

(a) Total capacitor voltage of the upper arm in phase A

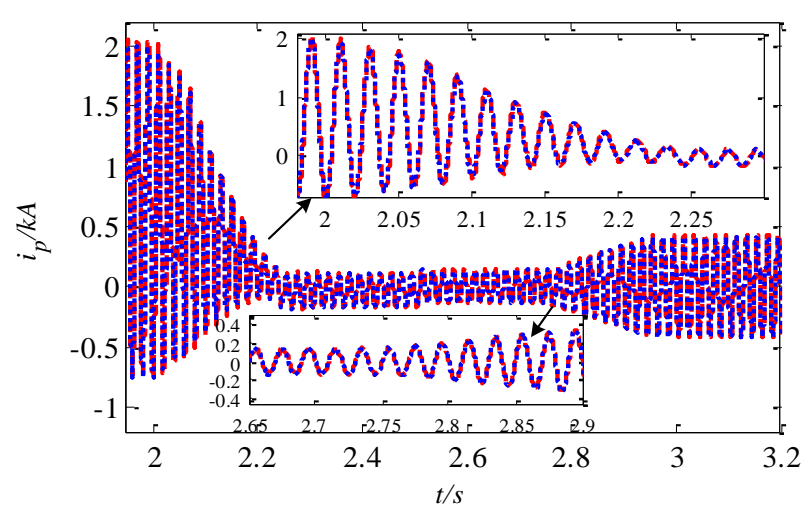

(b) Arm current of the upper arm in phase A 


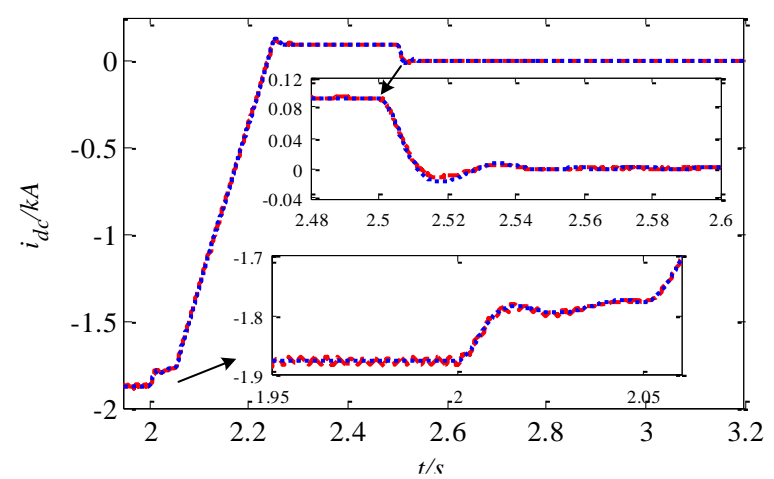

(c) The DC current

- - - Electromagnetic model …....... State-space model

Fig. 8 Verification of the state-space model

\section{ANALYSIS OF THE OPERATING ZONES}

The operating zones under different DC voltages are analyzed for the tested hybrid MMC. The considered operating constraints are listed in Table 2 . The DC voltage is set to increase from 0.01 p.u. to 0.5 p.u. with the step of 0.01 p.u.. The initial known conditions are selected as the DC current and the reactive power. In view of the operating constraints, the scanning range is set to be [-2 p.u., 2 p.u.] for the DC current with $I_{\mathrm{dc}, \text { step }}=0.005$ and [-1 p.u., 1 p.u.] for the reactive power with $Q_{\text {step }}=0.005$.

Table 2 Considered operating constraints

\begin{tabular}{l|l}
\hline Constraints & Values \\
\hline DC current & $\leq 2$ p.u. \\
sub-module voltage ripple & $\leq 13 \%$ \\
converter apparent power & $\leq 1$ p.u. \\
modulation ratio & $0 \sim 1.1$ \\
\hline
\end{tabular}

The calculated $P Q$ operating zones are depicted in Fig. 9 (a) in the form of 3D diagram. To make it easier to observe the edges of the $P Q$ operating zones under different $\mathrm{DC}$ terminal voltages, the $2 \mathrm{D}$ view is demonstrated in Fig. 9 (b). It can be inferred that under low DC terminal voltage, the feasible active power transmitted by the hybrid MMC is limited. On the contrary, since the $P Q$ operating zone can cover the whole range of $Q$-axis within $[-1,1]$, the converter's ability to transmit the reactive power is not influenced. 


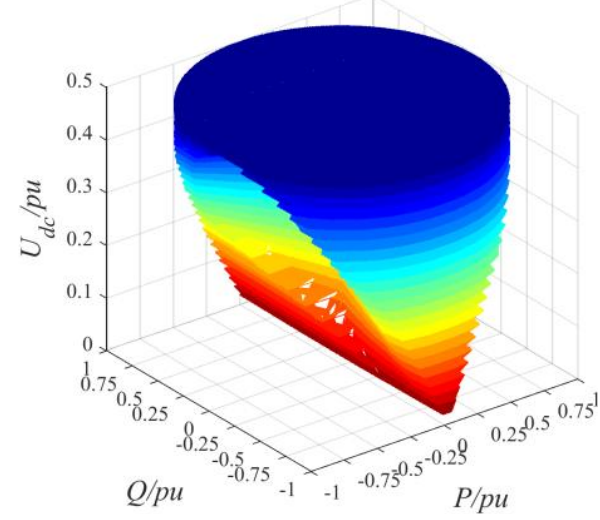

(a) $P Q-U_{\mathrm{dc}} 3 \mathrm{D}$ diagram

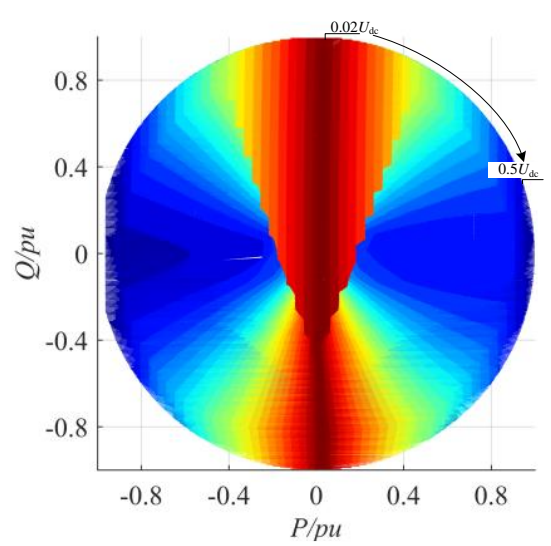

(b) $2 \mathrm{D}$ view

Fig. $9 P Q$ operating zone under different DC terminal voltages

\section{A. Analysis of PQ Operating Zones Under Different DC Terminal Voltages}

The calculated operating zones under $0,0.1,0.2$ and 0.5 p.u. DC voltage are depicted in Fig. 10 (a)-(d) respectively. Notice that the DC voltage is simulated as a controlled DC voltage source as depicted in Fig. 7. The active and reactive power at PCC is calculated. So when $U_{\mathrm{dc}}=0$, the active power at PCC equals to the power loss caused by the currents flowing through $R_{\text {arm }}$ and the line resistance.

In order to figure out how the operating constraints characterize the $P Q$ operating zone, the outlines of the operating zone characterized by each operating constraint are determined. By gathering all feasible operating points that respect certain operating constraint and exploring the boundary, the outline of the operating zone characterized by the corresponding operating constraint is obtained.

The calculated boundaries according to equations (23) and (33) are also shown in the Fig. 10 (b)-(c). It can be seen that the calculated boundaries are slightly different from the scanned boundaries, but they are similar to each other.

When $U_{\mathrm{dc}}=0,0.1$ and 0.2 p.u., as shown in Fig. 10 (a)-(c), the boundaries of the operating zone are determined by the HBSM balancing constraint, the sub-module capacitor voltage constraint, the DC current constraint and the MMC capacity constraint. 


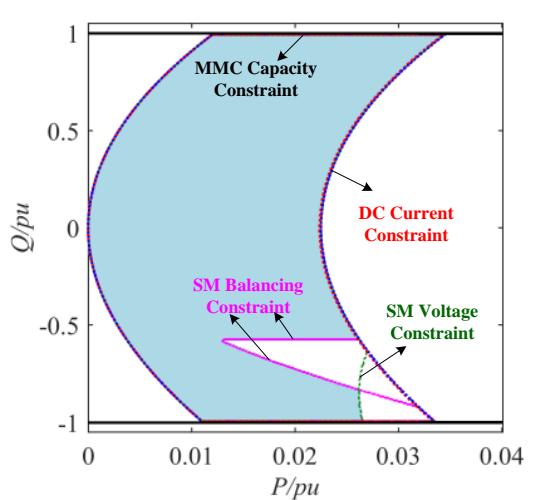

(a) $U_{\mathrm{dc}}=0$

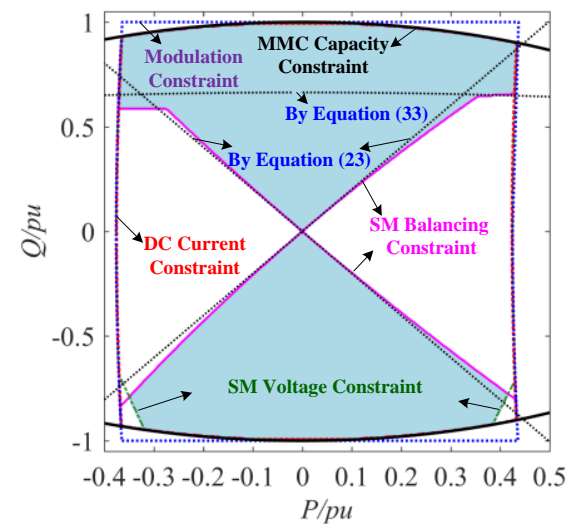

(c) $U_{\mathrm{dc}}=0.2$ p.u.

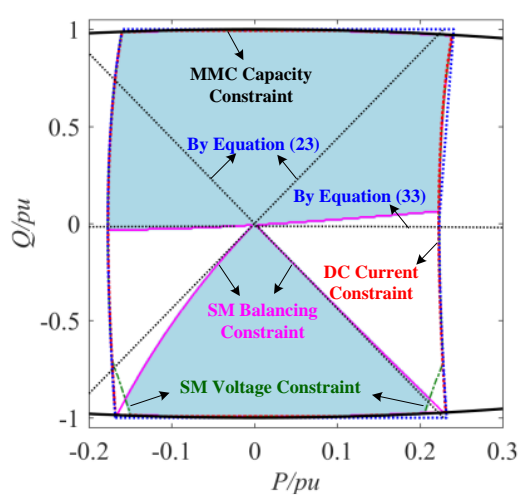

(b) $U_{\mathrm{dc}}=0.1$ p.u.

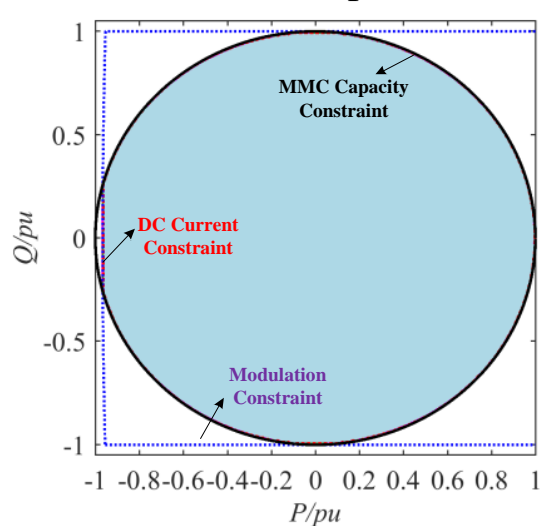

(d) $U_{\mathrm{dc}}=0.5$ p.u.

Fig. 10 The $P Q$ operating zones under different $D C$ voltages

As the DC terminal voltage continues to rise, as shown in Fig. 10 (d) where $U_{\mathrm{dc}}=0.5$ p.u., the feasible operating zone is limited by the converter capacity constraint and the DC current constraint only. Based on the parameters in Table $2, U_{\mathrm{dc}}=0.5 u_{\mathrm{dcN}}, U_{\mathrm{vN}}=0.55 u_{\mathrm{dcN}}$, so we can get $U_{\mathrm{dc}}>\sqrt{2} U_{\mathrm{vN}} / \sqrt{3} . i_{\mathrm{p}}$ is ensured to contain both positive and negative components, so the HBSM balancing constraint is satisfied naturally.

\section{B. Impact of Parameters on the Operating Zone}

The impact of converter parameters on the operating zone is calculated in this section. Taking the example of the scenario where $U_{\mathrm{dc}}=0.2$ p.u., the parameters to be analyzed include the arm inductance ( $\left.L_{\mathrm{arm}}\right)$, the sub-module capacitance $\left(C_{\mathrm{sub}}\right)$ and the FBSM ratio $\left(n_{\mathrm{FB}}\right)$. The parameters are varied to observe the change of the feasible operating zone, while the operating constraints remain unchanged. The results are shown in Fig. 11 (a)-(d). 


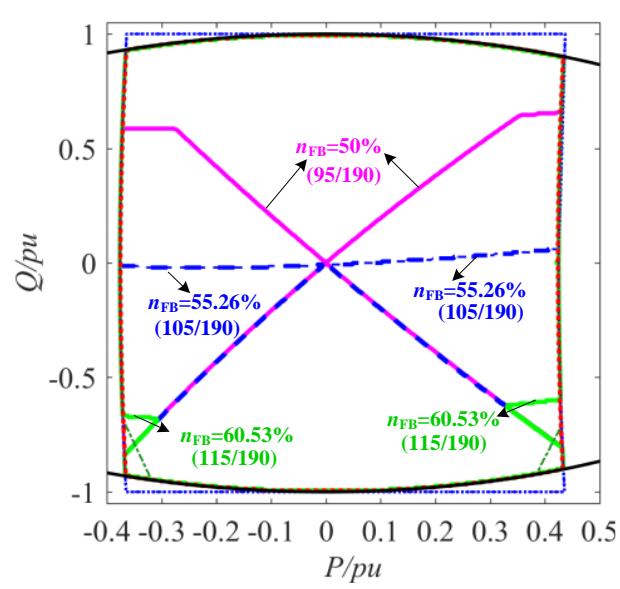

(a) FBSM ratio

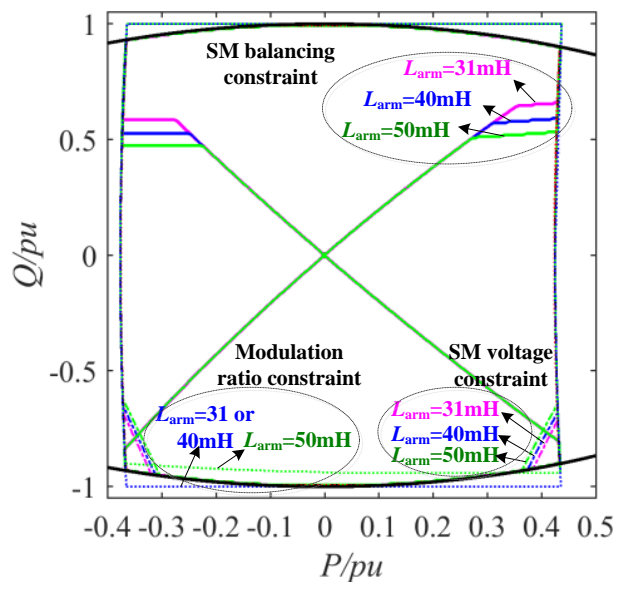

(c) arm inductance

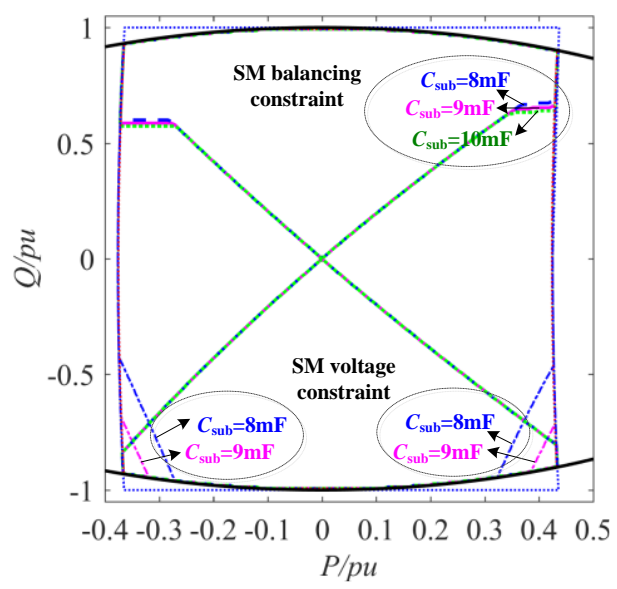

(b) sub-module capacitance
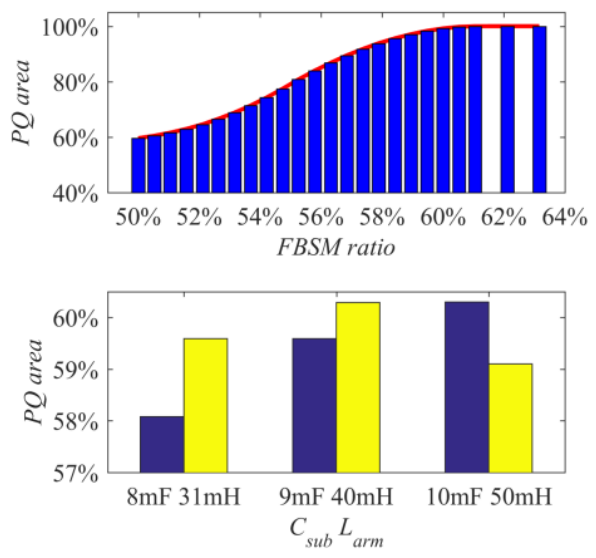

(d) summary

Fig. 11 Impact of various parameters on operating zone

As can be seen in Fig. 11 (a), the area of feasible operating zone increases obviously when $n_{\mathrm{FB}}$ increases from 50\% (95/190) to $60.53 \%$ (115/190). The restriction of the HBSM balancing constraint on the operating zone is attenuated with the rising of $n_{\mathrm{FB}}$.

In Fig. 11 (b), the sub-module capacitance is set to be $8 \mathrm{mF}, 9 \mathrm{mF}, 10 \mathrm{mF}$ separately. The operating zone slightly enlarges when $C_{\text {sub }}$ increases from $8 \mathrm{mF}$ to $10 \mathrm{mF}$. It can be inferred that the restriction of sub-module voltage constraint is weakened with the increase of capacitance. As in this case, when $C_{\text {sub }}=10 \mathrm{mF}$, the operating zone is no more limited by sub-module voltage constraint.

In Fig. 11 (c), the arm inductance is set to be $31 \mathrm{mH}, 40 \mathrm{mH}$ and $50 \mathrm{mH}$ respectively. It can be seen at the top of Fig. 11 (c) that the restriction regarding the HBSM balancing constraint is attenuated with the increase of $L_{\mathrm{arm}}$, and the operating zone enlarges. While the restriction regarding the modulation ratio constraint is reinforced when $L_{\mathrm{arm}}=50 \mathrm{mH}$, and the operating zone shrinks, as can be seen at the bottom of Fig. 11 (c). The operating zone determined by the sub-module voltage constraint also slightly 
shrinks with the increase of $L_{\mathrm{arm}}$.

Fig. 11 (d) summarizes the relation between the area of $P Q$ operating zone and the parameters quantitatively. When the ratio of FBSM reaches $61.05 \%(116 / 190)$, the area of $P Q$ operating zone is saturated and will not increase any more with the rise of the FBSM number. Compared to the FBSM ratio, the impact of the sub-module capacitance and the arm inductance on the area of $P Q$ operating zone is less.

\section{VII.CONCLUSIONS}

The eight-order SSTI state-space model of the hybrid MMC is proposed in this paper under rotating frames. The main intended application of the proposed SSTI state-space model is the connection with other sub-system models such as the AC system and the control system to describe the overall dynamics of a hybrid-MMC-based HVDC schemes for the eigenvalue-based small signal stability analysis. The proposed steady-state model can be applied to analytically analyze the operating characteristics and design feasible parameters satisfying multiple operating constraints.

To seek the boundaries of the feasible operating zone when multiple operating constraints are considered, an elaborated 'scan and check' method is proposed. Since the control effects such as sub-module sorting and balancing algorithm is assumed to be ideal, the calculation method provides useful information of the theoretically maximum operating zone. The efficient calculation method for approximated $P Q$ boundaries determined by the HBSM balancing constraint is derived from the perspective of qualified arm currents and arm voltages, which are respectively straight lines and circles with the slope, center and radius decided by the DC voltage. Besides, it is disclosed in this paper that when the DC terminal voltage is larger than the amplitude of the rated converter-side phase voltage, the HBSM balancing constraint is satisfied automatically.

The effects of different operating constraints on characterizing the $P Q$ operating zone are analyzed under different DC voltages. The impact of converter parameters on the operating zone is also presented. Compared to increasing the sub-module capacitance, increasing the FBSM ratio is more effective in expanding the $P Q$ operating zone, since the HBSM balancing constraint is dominant under low DC 
terminal voltage.

\section{ACKNOWLEDGEMENT}

This work was supported by the National key research and development program of China (2016YFB0901002) and the Initiative Postdocs Supporting Program (No. BX201700088).

\section{REFERENCES}

[1] A. Lesnicar, R. Marquardt, "An innovative modular multilevel converter topology suitable for a wide power range," Proc. Power Tech Conf., Bologna, Italy, 2003.

[2] X. Li, Q. Song, W. Liu, et al, “Zero-sequence voltage injection control scheme of modular multilevel converter supplying passive networks under unbalanced load conditions," Electr. Power Syst. Res., vol. 121, pp. 270-278, April 2015.

[3] H. Saad, J. Mahseredjian, S. Dennetiere, et al, "Interactions studies of HVDC-MMC link embedded in an AC grid," Electr. Power Syst. Res., vol. 138, pp. 202-209, Sep. 2016.

[4] J. Zhang, C. Zhao, "Analysis and control of MMC-HVDC under unbalanced voltage conditions," Electr. Power Syst. Res., vol. 140, pp. 528-538, Nov. 2016.

[5] J. Li, G. Konstantinou, H.R. Wickramasinghe, et al, "Investigation of MMC-HVDC operating region by circulating current control under grid imbalances," Electr. Power Syst. Res., vol. 152, pp. 211-222, Nov. 2017.

[6] E. Prieto-Araujo, A. Junyent-Ferre, C. Collados-Rodriguez, et al, "Control design of Modular Multilevel Converters in normal and AC fault conditions for HVDC grids," Electr. Power Syst. Res., vol. 152 , pp. 424-437, Nov. 2017.

[7] R. Li, J.E. Fletcher. "A novel MMC control scheme to increase the DC voltage in HVDC transmission systems,” Electr. Power Syst. Res., vol. 143, pp. 544-553, Feb. 2017.

[8] R. Zeng, L. Xu, L. Yao, et al. "Design and operation of a hybrid modular multilevel converter". IEEE Trans. Power Electron., vol. 30, no. 3, pp. 1137-1146, March 2015

[9] R. Zeng, L. Xu, L. Yao, et al. "Precharging and DC fault ride-through of hybrid MMC-based HVDC systems”. IEEE Trans. Power Del., vol. 30, no. 3, pp. 1298-1306, June 2015. 
[10]W. Lin, D. Jovcic, S. Nguefe, et al, "Full-bridge MMC converter optimal design to HVDC operational requirements," IEEE Trans. Power Del., vol. 31, no. 3, pp. 1342-1350, June 2016.

[11]V. Hofmann and M. M. Bakran, "Optimized design of a hybrid-MMC and evaluation of different MMC topologies," Proc. 18th Euro. Conf. Power Electron. Appl., Karlsruhe, Germany, 2016, pp. $1-9$.

[12]S. Cui and S. K. Sul. "A Comprehensive DC short-circuit fault ride through strategy of hybrid modular multilevel converters (MMCs) for overhead line transmission”. IEEE Trans. Power Electron., vol. 31, no. 11, pp. 7780-7796, Nov. 2016.

[13]J. Hu, K. Xu, L. Lin, et al. “Analysis and enhanced control of hybrid-MMC-based HVDC Systems during asymmetrical DC voltage faults”. IEEE Trans. Power Del., vol. 32, no. 3, pp. 1394-1403, June 2017.

[14]W. Xiang, W. Lin, L. Xu, J. Wen, "Enhanced independent pole control of hybrid MMC-HVDC system," IEEE Trans. Power Del., (early access)

[15]G. Bergna, J. A. Suul and S. D'Arco, "State-space modelling of modular multilevel converters for constant variables in steady-state," Proc. 17th Workshop Control Model. Power Electron., Trondheim, Norway, 2016, pp. 1-9.

[16]D. Jovcic and A. A. Jamshidifar, "Phasor Model of Modular Multilevel Converter With Circulating Current Suppression Control," IEEE Trans. Power Del., vol. 30, no. 4, pp. 1889-1897, Aug. 2015.

[17]Xiaojun Lu, W. Lin, Jinyu Wen, et al, "Dynamic phasor modelling and operating characteristic analysis of half-bridge MMC," Proc. 8th Int. Power Electron. Motion Control Conf., Hefei, China, 2016.

[18]A. Antonopoulos, L. Angquist and H. P. Nee, "On dynamics and voltage control of the Modular Multilevel Converter," Proc. 13th Eur. Conf. Power Electro. Applicat, Barcelona, Spain, 2009.

[19]W. Xiang, W. Lin, T. An, J. Wen and Y. Wu, "Equivalent electromagnetic transient simulation model and fast recovery control of overhead VSC-HVDC based on SB-MMC," IEEE Trans. Power Del., vol. 32, no. 2, pp. 778-788, April 2017. 